\title{
Séroprévalence et évolution de la sérologie toxoplasmique chez les transplantés rénaux, hépatiques et greffés de moelle osseuse
}

\section{Seroprevalence and evolution of toxoplasmic serology in renal, hepatic and bone marrow transplant recipients}

\author{
Guechi $\mathrm{N}^{1}$, Ghaffor $\mathrm{Y}^{1}$,Oukachbi $\mathrm{S}^{1}$,Hamrioui $\mathrm{B}^{1}$ \\ 1- Laboratoire de Parasitologie-Mycologie de l'hôpital Mustapha -Pacha d' Alger (Algérie)
}

Auteur correspondant: Naila Guechi, Maître de conférences (A) en Parasitologie-Mycologie au Laboratoire de Parasitologie-Mycologie du CHU Mustapha Pacha d'Alger (Algérie) Email: guechinaila@ hotmail.fr

\section{Résumé:}

Objectifs : Le but de notre étude était d'évaluer la séroprévalence ainsi que le risque de toxoplasmose aiguë chez des patients qui ont subi une transplantation rénale, hépatique ou une greffe de moelle osseuse.

Patients et méthode : Une étude prospective a été menée du 01 Janvier 1999 à Décembre 201.Les sérologies toxoplasmiques ont été réalisées à partir des sérums de 888 patients (444 couples donneurs/receveurs) au niveau du laboratoire de Parasitologie -Mycologie du CHU Mustapha d'Alger par la technique immunoenzymatique ELISA (358 candidats à la transplantation rénale, 69 à la greffe de moelle osseuse, 17 à la transplantation hépatique).

Résultats: La séroprévalence globale était de 54,5\%.81 receveurs de transplantation rénale ont été transplantés et avaient des sérologies régulières de toxoplasmose :45 étaient séropositifs et 36 séronégatifs avant la transplantation ; aucune toxoplasmose aiguë n'a été observée chez les 36 patients séronégatif et aucune réactivation chez les 45 autres séropositifs après la transplantation .17 receveurs de transplantation hépatique ont été transplantés et avaient des sérologies régulières de toxoplasmose:5 était séropositifs et 5 séronégatif avant la transplantation; aucune toxoplasmose aiguë n'a été observée chez les 5 patients séronégatifs et aucune réactivation chez les 5 autres séropositifs après la transplantation. Pour les greffés de moelle osseuse, pour des raisons pratiques, le suivi sérologique n'a pu être effectué.

Conclusion: La surveillance sérologique chez les transplantés reste nécessaire surtout chez les séronégatifs. II est important de connaître la sérologie du receveur avant la transplantation et si possible celle du donneur afin d'interpréter plus facilement toute modification. Cette surveillance doit être très régulière pour permettre le diagnostic rapide d'une toxoplasmose évolutive.

Mots-clés : Séroprévalence, Suivi sérologique, Toxoplasmose, Transplanté.

\section{Abstract}

Objectives: The aim of our study was to assess the seroprevalence as well as the risk of acute toxoplasmosis in patients who have undergone kidney, liver or bone marrow transplants.

Patients and method: A prospective study was carried out from January 01, 1999 to December 2017. Toxoplasmic serologies were carried out from the serum of 888 (444 donor / recipient couples) patients at the level of the Parasitology-Mycology laboratory of the Mustapha University Hospital. 'Algiers by the immunoenzymatic ELISA technique (358 candidates for renal transplantation, 69 for bone marrow transplant, 17 for liver transplantation).

Results: The overall seroprevalence was 54.5\%. 81 kidney transplant recipients were transplanted and had regular toxoplasmosis serologies: 45 were seropositive and 36 seronegative before transplantation: no acute toxoplasmosis was observed in the 36 patients and no reactivation in the remaining 45 after transplantation. 17 liver transplant recipients were transplanted and had regular toxoplasmosis serologies, 5 were seropositive and 5 seronegative before transplantation: no acute toxoplasmosis was observed in the 5 seronegative patients and no reactivation in the remaining 5 after transplantation. For bone marrow transplant recipients, for practical reasons, serological monitoring could not be carried out.

Conclusion: serological surveillance in transplant recipients remains necessary, especially in seronegative patients. It is important to know the serology of the recipient before the transplant and if possible that of the donor in order to more easily interpret any changes. This monitoring must be very regular to allow the rapid diagnosis of progressive toxoplasmosis.

Keywords: Seroprevalence, Serological monitoring, Toxoplasmosis, Transplanted. 


\section{Introduction:}

La toxoplasmose est une parasitose souvent asymptomatique chez l'immunocompétent mais peut être responsable de formes sévères chez l'immunodéprimé dont le greffé. Le transplanté peut développer une toxoplasmose aiguë par 3 voies : transmission par le greffon, réactivation d'une infection ancienne $\mathrm{du}$ fait de son traitement immunosuppresseur ou infection acquise après la greffe [1]. Bien que la toxoplasmose reste rare chez les greffés, la mortalité chez cette population reste le plus souvent élevée [2,3]. Des mesures préventives doivent donc être utilisées si le receveur n'est pas immunisé consistant en des conseils hygiéno-diététiques identiques à ceux fournis à la femme enceinte ainsi qu'un suivi sérologique chez le receveur après transplantation [1]. En Algérie depuis plusieurs années, le nombre de greffes ne cesse d'augmenter, plusieurs types de greffes sont alors pratiquées, moelle osseuse, transplantation rénale ou encore transplantation hépatique et parmi les bilans prégreffe demandé, la sérologie toxoplasmique. Le but de notre travail est d'évaluer la séroprévalence de la toxoplasmose chez les donneurs et les candidats à une transplantation et de voir l'évolution de la toxoplasmose chez ces derniers en se basant sur les résultats des prélèvements reçu au laboratoire.

\section{Matériel et méthodes :}

Population : Il s'agit d'une étude prospective qui s'est déroulée de Janvier 1999 à Décembre 2017; durant cette période des prélèvements de 888 patients (444 couples donneurs /receveurs) sont parvenus au niveau de notre laboratoire pour une sérologie toxoplasmique dans le cadre d'un bilan prégreffe ou d'un suivi après greffe demandé par leur médecin. La répartition de ces 888 patients s'est effectuée comme suit: 358 couples candidats à la transplantation rénale, 69 à la greffe de moelle osseuse, 17 à la transplantation hépatique; la majorité des prélèvements parvenaient des différents services du CHU Mustapha Pacha d'Alger, d'autres du CHU Nafissa Hammoud et du CHU de Béni Messous.

Méthode : Les prélèvements ont été effectués par ponction veineuse par une infirmière qualifiée après avoir rempli une fiche de renseignement; les prélèvements de sang total étaient étiquetés , centrifugés à 3000 tour/min pendant 5 à 10 min afin d'obtenir le sérum, puis numérotés et conservés à + $4^{\circ} \mathrm{c}$ pendant une semaine au maximum puis à $20^{\circ} \mathrm{C}$. Les sérologies toxoplasmiques ont été réalisées à pâtir des sérums des donneurs et des receveurs par la technique immunoenzymatique ELISA ( Platelia TM Toxo IgG, IgM BIORAD®(72840) pour la recherche des IgG et des IgM antitoxoplasmiques dont le seuil de positivité est supérieur ou égale à $10 \mathrm{UI} / \mathrm{ml}$ pour les IgG, un ratio $\geq 0,1$ est considéré comme positif pour les IgM. Le test d'avidité (Platelia ${ }^{\mathrm{TM}}$ ToxoIg GAVIDITY) (72842) a été pratiqué chez les patients ayant des IgM positifs afin de dater l'infection toxoplasmique, un test d'avidité est considéré comme positif si il est $\geq 0,4$ (infection de plus de 05 mois). Le suivi post greffe a été réalisé pour certains patients par ELISA.

Suivi des patients: pour les transplantés rénaux et hépatiques, nous avons demandé aux receveurs un premier prélèvement pratiqué pour une sérologie toxoplasmique 7jours avant la transplantation puis un suivi sérologique mensuel après transplantation allant du 30ème au 450ème jour quel que soit leur statut sérologique.

\section{Résultats :}

\section{Résultats de la séroprévalence :}

L'âge des patients variait de 4 à 64 ans pour la greffe de moelle osseuse, de 5 à 67 ans pour la transplantation rénale et de 24 à 58 ans pour la transplantation hépatique. La moyenne d'âge pour l'ensemble de la population était de 35.29 ans.Les statuts sérologiques des 444 couples selon le type de greffe sont reportés sur le tableau I.

Tableau I: Statuts sérologiques des 444 couples selon le type de greffe

\begin{tabular}{lcccc}
\hline & \multicolumn{4}{c}{ Statuts sérologiques } \\
Type de greffe/ & $\mathrm{R}+/ \mathrm{D}-$ & $\mathrm{R}+/ \mathrm{D}$ & $\mathrm{R}-/ \mathrm{D}$ & $\mathrm{R}-/ \mathrm{D}$ \\
Transplantation & & + & + & - \\
\hline Rénale & 70 & 126 & 79 & 83 \\
Moelle osseuse & 20 & 16 & 12 & 21 \\
Hépatique & 4 & 5 & 5 & 3 \\
Total & 94 & 147 & 96 & 107 \\
\hline
\end{tabular}

$R=$ Receveur $\quad D=$ Donneur

Les statuts sérologiques détaillés des 358 couples candidats à la transplantation rénale sont ci-bas.

Tableau II: Statuts sérologiques détaillés des 358 couples candidats à la transplantation rénale

\begin{tabular}{lll}
$\begin{array}{c}\text { Statuts } \\
\text { sérologiques }\end{array}$ & \multicolumn{1}{c}{ Statuts sérologiques détaillés par } \\
ELISA
\end{tabular}

Ceux des 69 à la greffe de moelle osseuse et les 17 à la transplantation hépatique sont reportés respectivement sur le tableau III et IV. 
Tableau III: Statuts sérologiques détaillés des 69 couples candidats à la greffe de moelle osseuse

\begin{tabular}{|c|c|c|}
\hline $\begin{array}{c}\text { Statuts } \\
\text { sérologiques }\end{array}$ & \multicolumn{2}{|c|}{$\begin{array}{l}\text { Statuts sérologiques détaillés par } \\
\text { ELISA }\end{array}$} \\
\hline $20 \mathrm{R}(+) / \mathrm{D}$ & $20 \mathrm{R} \operatorname{IgG}(+) / \operatorname{IgM}(-)$ & - \\
\hline$(-)$ & 20 D IgG (-) / IgM(-) & \\
\hline $16 \mathrm{R}(+) / \mathrm{D}$ & 13R $\operatorname{IgG}(+) / \operatorname{IgM}(-)$ & $3 \mathrm{R} \mathrm{IgG(+)} \mathrm{/}$ \\
\hline$(+)$ & 13D $\operatorname{IgG}(+) / \operatorname{IgM}(-)$ & $\begin{array}{l}\operatorname{IgM}(+) \\
3 \mathrm{D} \mathrm{IgG(}(+) / \\
\operatorname{IgM}(-)\end{array}$ \\
\hline $12 \mathrm{R}(-) /$ & 11R $\operatorname{IgG}(-) / \operatorname{IgM}(-)$ & 1 R IgG(-) \\
\hline $\mathrm{D}(+)$ & $11 \mathrm{D} \operatorname{IgG}(+) / \operatorname{IgM}(-)$ & $\begin{array}{l}/ \operatorname{IgM}(-) \\
1 \mathrm{D} \operatorname{IgG}(+) \\
/ \operatorname{IgM}(+)\end{array}$ \\
\hline $\begin{array}{l}21 \mathrm{R}(-) / \\
\mathrm{D}(-)\end{array}$ & $\begin{array}{l}\text { 21RIgG (-)/IgM(-) } \\
21 \mathrm{DIgG}(-) / \operatorname{IgM}(-)\end{array}$ & - \\
\hline
\end{tabular}

$$
R=\text { Receveur } \quad D=\text { Donneur }
$$

Tableau IV: Statuts sérologiques détaillés des 17 couples candidats à la transplantation hépatique :

\begin{tabular}{|c|c|c|}
\hline $\begin{array}{c}\text { Statuts } \\
\text { sérologiques }\end{array}$ & \multicolumn{2}{|c|}{$\begin{array}{c}\text { Statuts sérologiques détaillés par } \\
\text { ELISA }\end{array}$} \\
\hline & $4 \mathrm{R} \operatorname{IgG}(+) / \operatorname{IgM}(-)$ & - \\
\hline \multirow[t]{2}{*}{$4 \mathrm{R}(+) / \mathrm{D}(-)$} & 4 D IgG (-) / IgM(-) & \\
\hline & $5 \mathrm{R} \operatorname{IgG}(+) / \operatorname{IgM}(-)$ & - \\
\hline \multirow[t]{2}{*}{$5 \mathrm{R}(+) / \mathrm{D}(+)$} & $5 \mathrm{D} \operatorname{IgG}(+) / \operatorname{IgM}(-)$ & \\
\hline & 5R IgG (-) / IgM(-) & - \\
\hline $5 \mathrm{R}(-) / \mathrm{D}(+)$ & $5 \mathrm{D} \operatorname{IgG}(+) / \operatorname{IgM}(-)$ & \\
\hline \multirow[t]{2}{*}{$3 \mathrm{R}(-) / \mathrm{D}(-)$} & 3RIgG (-) / IgM(-) & - \\
\hline & 3DIgG (-) / IgM(-) & \\
\hline
\end{tabular}

Les séroprévalences retrouvées ont été comme suit: au total $54,5 \%$ des patients étaient séropositifs dont $54,2 \%$ chez les receveurs et $54,7 \%$ chez les donneurs. Une sérologie positive a été retrouvée chez $56 \%$ pour la transplantation rénale $(54,7 \%$ chez les receveurs, $57,2 \%$ chez les donneurs), $46,3 \%$ pour la greffe de moelle osseuse $(52,1 \%$ chez les receveurs, $40,5 \% \mathrm{chez}$ les donneurs) et $55,8 \%$ pour la transplantation hépatique $(52,9 \%$ chez les receveurs, $58,8 \%$ chez les donneurs). Sur les 888 patients, 21 ont eu des IgM positifs par ELISA et ont bénéficié du test d'avidité afin de dater l'infection toxoplasmique: pour tous les patients l'indice d'avidité était supérieur à 0,4 concluant à une infection datant de plus de 05 mois.

Devenir des patients :

Pour les candidats à la transplantation rénale : 90 ont été greffés dont 81 suivis, 6 perdus de vue et 3 décédés ( 2 post greffe dont la cause n'est pas connue et un par une pneumopathie au 90ème jour).

Pour les candidats à transplantation hépatique : 12 ont été greffés dont 10 suivis, 2 perdus de vue.

Pour les candidats à la greffe de moelle osseuse: 33 ont été greffés et n'ont pas pu être suivi.

Résultats des suivis :

Pour les séronégatifs (aussi bien chez les receveurs que chez les donneurs), des conseils hygièno- diététiques à suivre ont été donnés comme ceux fournis aux femmes enceintes par écrit.

Pour les greffés de moelle osseuse, la présence de kystes de Toxoplasma gondii chez le receveur et l'absence d'anticorps dans la moelle du donneur a préconisé une chimioprophylaxie chez les receveurs.Pour les transplantés rénaux: aucune séroconversion n'a été observée chez les 35receveurs séronégatif et aucune réactivation sérologique chez les 45 receveurs séropositifs .Pour les transplantés hépatiques: aucune séroconversion n'a été observée chez les 5 receveurs séronégatif et aucune réactivation chez les 5 receveurs séropositifs. Pour les greffés de moelle osseuse: aucun suivi n'a été fait pour des raisons pratiques .Les résultats comparatifs entre les transplantés rénaux hépatiques sont illustrés dans le tableau V.

Tableau V: Tableau comparatif entre les transplantés rénaux, hépatiques et greffés se moelle osseuse

\begin{tabular}{|c|c|c|c|}
\hline $\begin{array}{l}\text { Type de greffe ou } \\
\text { Transplanta- } \\
\text { Résultats }\end{array}$ & Rénale & Hépatique & $\begin{array}{l}\text { Moelle } \\
\text { osseuse }\end{array}$ \\
\hline Nombre de couples & 358 & 17 & 69 \\
\hline $\begin{array}{r}\text { Séroprévalence : D } \\
\mathrm{R}\end{array}$ & $\begin{array}{l}57,2 \% \\
54,7 \%\end{array}$ & $\begin{array}{l}58,8 \% \\
52,9 \%\end{array}$ & $\begin{array}{l}40,5 \% \\
52,7 \%\end{array}$ \\
\hline $\begin{array}{l}\text { Nombre de patients } \\
\text { transplantés ou } \\
\text { greffés }\end{array}$ & 90 & 12 & 33 \\
\hline $\begin{array}{l}\text { Nombre de receveurs } \\
\text { suivis après } \\
\text { transplantation ou } \\
\text { greffe }\end{array}$ & 81 & 10 & Aucun \\
\hline $\begin{array}{l}\text { Nombre de } \\
\text { toxoplasmose aiguë } \\
\text { ou de réactivation }\end{array}$ & Aucun & Aucun & - \\
\hline
\end{tabular}

\section{Discussion}

La toxoplasmose est une infection opportuniste redoutée aussi bien chez les greffés de moelle osseuse que les transplantés d'organes solides. Dans le cas de la greffe, lorsque le receveur est séronégatif et le donneur séropositif, la transmission du toxoplasme par le greffon est possible et concerne essentiellement la transplantation d'organe solide. Chez les greffés, en plus de la primo infection, la toxoplasmose peut se manifester suite à une réactivation de kystes latents du fait du traitement immunosuppresseur [4]. Une étude du suivi des greffés rénaux sur 3ans par Iqbal en 2003a rapporté qu'une réactivation étaitsurvenue chez $9.7 \%$ des patients alors que les cas de primo infection n'ont concerné que $2.7 \%$ des receveurs [5]. Durant ce travail, bien qu'aucun cas de toxoplasmose maladie n'a été diagnostiqué, 3 décès ont été enregistréchez des 
greffés rénaux ( 2 post greffe dont la cause n'a pas été connue et le $3^{\text {ème }}$ à $\mathrm{J} 90$ post greffe par une pneumopathie).Lors de transplantation d'organes solides, la période la plus à risque d'une infection toxoplasmique est liée au degré d'immunodépression, elle apparaît le plus souvent dans les trois mois suivant la greffe [4]. Concernant les greffés de moelle osseuse, le risque de réactivation toxoplasmique chez les patients séropositifs est particulièrement élevé durant les 2 à 4 mois suivant la greffe; cette période peut être prolongée de 6 mois voire plus sachant qu'en moyenne, il faut 2 à 5 ans pour obtenir une bonne récupération des fonctions lymphocytaires T [6]. Dans notre étude la séroprévalence était de $54,5 \%$, résultat similaire à celui retrouvé en Malaisie en 2010 avec $56,7 \%$ [7], et supérieur à celui retrouvé en Iran en 2014 avec50\%, [8].Notre séroprévalence chez des candidats à la greffe rénale a étésupérieure à celle retrouvée en Égypte en 2012 avec 38.3\% [8],et inférieure à celle retrouvée en Turquie en 2005[10]. Chez les greffés de moelle osseuse, la séroprévalence a été de $46,3 \%$ avec une séroprévalence de $52,1 \%$ chez les receveurs.Pour certains auteurs, la présence d'anticorps anti toxoplamiques est considérée comme un risque majeur d'infection chez les greffés, ceci s'applique surtout pour les greffés de moelle osseuse, de plus ce risque semble s'atténuer chez les receveurs lorsque le donneur est séropositif [8]. Chez les greffés de moelle osseuse, le risque de transmission par le greffon reste relativement très rare, ainsi le cas de figure le plus à risque est celui de la réactivation toxoplasmique chez les receveurs séropositifs, ce risque de réactivation serait plus fréquent chez les receveurs de donneurs séronégatifs que les receveurs de donneurs séropositifs.Néanmoins, aucun consensus n'existe et les protocoles varient en fonction des régions, ainsi aux Pays Bas, vue la très faible incidence de la toxoplasmose chez les greffés rénaux, aucune sérologie toxoplasmique n'est effectuée en pré greffe [11], alors qu'en France la sérologie toxoplasmique fait partie du bilan pré greffe habituel. La présence des IgM spécifiques peut faire craindre une infection récente chez le donneur; le risque pour le receveur est que le toxoplasme soit transmis par le greffon ou le sang du greffon dans les deux cas il peut alors y avoir dissémination du fait de l'immunodépression. Au cours de notre étude, 21 patients étaient positifs en IgM:17 patients pour la transplantation rénale dont 10 receveurs et 07 donneurs .04 patients pour la greffe de moelle osseuse dont 02 receveurs et 02 donneurs .Afin de dater l'infection toxoplasmique chez ces patients, un test d'avidité a été effectué (certains auteurs recommandent également de réaliser une PCR Toxoplasma gondii) [12];la présence d'IgM en pré greffe n'a pas interféré dans la programmation ou non de la greffe car présentant un indice d'avidité élevé, supérieur à 0.4 ; néanmoins selon la littérature, la présence chez les donneurs d'IgM spécifiques ne constitue pas une raison pour le report de la greffe mais le receveur bénéficiera d'une surveillance clinique et biologique rapprochée [13]. Pour certains auteurs, un traitement prophylactique de 6 semaines peut être considéré comme une alternative chez les receveurs d'un rein issu d'un donneur IgM positif [9]. 81 receveurs de transplantation rénale ont bénéficiéd'un suivi sérologique mensuel allant de 30 à 450 jours, aucune séroconversion n'a été observée chez les 35 receveurs séronégatif et aucune réactivation sérologique n'a été observée chez les 45 receveurs séropositifs. Alors que pour les greffés de moelle osseuse, pour des raisons pratiques, le suivi sérologique n'a pu être effectué. Sur les 17 receveurs de transplantation hépatique qui ont bénéficié d'un suivi sérologique mensuel allant de 30 à 450 jours, aucune séroconversion n'a été observée chez les 5 receveurs séronégatifs et aucune réactivation sérologique n'a étéobservée chez les 5receveurs séropositifs. Bien que la toxoplasmose soit relativement rare chez les greffés, le taux de mortalité de cette infection chez cette population reste très élevée, pouvant atteindre les $65 \%$ pour la transplantation rénale [4]. Le retard du diagnostic et de la prise en charge thérapeutique peut expliquer la mortalité élevée de cette infection ; les symptômes sont souvent non spécifiques, les patients présentent généralement des troubles pulmonaires [11], fièvre (dans $80 \%$ des cas) et malaise peuvent également être retrouvés; de plus la présence d'infection concomitante n'est pas rare, tout cela participe au retard diagnostic [4]. Le diagnostic peut être posé par l'isolement du parasite au niveau de produit biologique tel que le LBA, LCR ou biopsie, ou via une sérologie ou encore par des techniques de biologie moléculaire, néanmoins les performances de ces méthodes restent faibles [4]. Ainsi le meilleur moyen de lutter contre cette infection reste la prévention en associant chimioprophylaxie et surveillance clinique et biologique.Comme perspective, nous souhaiterons une réglementation algérienne basée sur un décret comme celui fait en France et qui impose le dépistage de la toxoplasmose chez le donneur pour tout prélèvement d'organe ou de cellules d'origine humaine conformément à l'article 3 de l'arrêté du 23 décembre 2010 (pris en application de l'article R. 1211-16 du Code de la santé publique). Ce texte précise que les analyses peuvent être réalisées en différé, dans les heures suivant le prélèvement, sans que l'absence de leur résultat ne fasse obstacle à la réalisation de la greffe. Un consensus doit être établit encadrant la prophylaxie de la toxoplasmose chez les greffés, car en Algérie les greffés reçoivent uniquement du Bactrim pendant les 3 à 6 mois suivant la greffe, ce fut le cas dans cette étude, bien que ce traitement est surtout prescrit en prévention de la pneumocystose mais il est également efficace contre la toxoplasmose.

\section{Conclusion}

La toxoplasmose fait partie des infections opportunistes les plus sévères touchant les greffés.Une vigilance est nécessaire devant l'association d'une séroprévalence de plus de $50 \%$ et l'immunodépression de cette population. Devant une clinique atypique, le diagnostic de la toxoplasmose est très souvent retardé 
donnant ainsi un taux de mortalité élevé. Afin de pallier à cela, une mise à jour des connaissances en matière de la pathogénicité, de procédures d'identification des facteurs de risque et des mesures de prévention de la toxoplasmose chez cette population à risque est nécessaire. En Algérie, la greffe étant en nette progression, une coopération cliniciensbiologistes est indispensable à l'élaboration d'une démarche consensuelle que ce soit sur le plan diagnostic que prophylactique en accord avec l'épidémiologie locale de cette infection et les moyens techniques disponibles dans notre pays.

\section{Conflit d'intérêt}

Les auteurs déclarent n'avoir aucun conflit d'intérêt.

\section{Références}

1) Derouin F. Parasitoses des immunodéprimés. Rev. Prat $200157: 167-173$

2) Vogelzang JL, van Stralen KJ, Noordzij M, et al. Mortality from infections and malignancies in patients treated with renal replacement therapy: data from the ERA-EDTA registry. Nephrol Dial Transplant 2015 (30):1028-37.

3) Linares L, Cofán F, Cervera $\mathrm{C}$, et al. Infectionrelated mortality in a large cohort of renal transplant recipients. Transplant Proc 2007 (39):2225-7.

4) Clissold R, Coralie B. Late presentation of toxoplasmosis in renal transplant recipients, NDT Plus 2010 (3): $480-482$.

5) Iqbal J,. Nampoory M.R.N, Johnv K.V., Khalid N, and Al-Mousawi M. Determination of Antibodies to Toxoplasma Gondii and CMV in Renal Transplant
Recipients. Transplantation Proc,2003 (35) : 27032705.

6) Delcher L. Impact de l'utilisation des médicaments génériques en post greffe de moelle osseuse. [Thèse de doctorat].[Grenoble]:université Joseph Fourier ;2014 . 7) Veeranoot N, Teoh HL, Rogan L, Imit I, Jamaiah I and Tan SY. Seropepidemiology of toxoplasmosis in renal patients, Southeast Asian J Trop Med Public Health,2010 (42);2:.

8) Ahmadpour E. Toxoplasmosis in immunocompromised patients in Iran. J Infect Dev Ctries, 2014, 8(12):1503-1510.

9) Sullivan W\&V Jeffers V. Mechanisms of Toxoplasma gondii persistence and latency. FEMS Microbiol Rev 2012, 36(3): 717-733.

10) Ocak S, Duran N, Eskiocak AF, Aytac H. AntiToxoplasma gondii antibodies in hemodialysis patients receiving long-term hemodialysis therapy in Turkey. Saudi Med J 2005(26): 1378-82.

11) Wulf MWH, Van Crevel R, Portier R, ter Meulen CG, Melchers WJG, van der Ven A and Galama JMD, Toxoplasmosis after Renal Transplantation: Implications of a Missed Diagnosis. J Clin Microbiol, 2005, 43 (7): 3544-3547.

12) Duléry R, Giraud C, Beaumont JL, Bilger K, Borel $\mathrm{C}$, Dhedin $\mathrm{N}$ et al. How to handle unexpected biological abnormalities observed in the pre-donation workup for hematopoietic stem cell transplantation: An SFGM-TC report on pre-transplant cytomegalovirus, Epstein-Barr virus, Toxoplasma gondii, or syphilis IgM positive serology test. Pathol Biol 2013, 61(4):158-9.

13) Renoult E,Georges E, Biava MF, Hulin C,Frimat L, Hestin D, et al. Toxoplasmosis in Kidney Transplant Recipients: A Life-Threatening but Treatable Disease. Transplantation Proc,1997,( 29): 821-822. 